\title{
MALOCLUSIONES EN NIÑOS Y ADOLESCENTES DE CASERÍOS Y COMUNIDADES NATIVAS DE LA AMAZONÍA DE UCAYALI, PERÚ
}

\author{
Arón Aliaga-Del Castillo1,a, Manuel Antonio Mattos-Vela 2,a,b, Rosalinda Aliaga-Del Castillo 3 ,a,c, \\ Claudia Del Castillo-Mendoza ${ }^{4, a}$
}

\begin{abstract}
RESUMEN
Se realizó un estudio descriptivo transversal para evaluar la prevalencia de maloclusiones en niños y adolescentes de 2 a 18 años de edad de caseríos y comunidades nativas de la selva de Ucayali, Perú. Se evaluó la presencia de maloclusiones usando la clasificación de Angle así como alteraciones ortodónticas. Se incluyeron 201 sujetos, 106 $(52,7 \%)$ fueron mujeres, la mayoría $(54,7 \%)$ tuvieron entre 6 y 12 años. Se encontró una prevalencia de maloclusiones del 85,6\%; la más prevalente según la clasificación de Angle fue la clase I (59,6\%). Se evidenciaron alteraciones ortodónticas en el $67,2 \%$ de casos. Las alteraciones ortodónticas encontradas más frecuentes fueron apiñamiento dentario $(28,4 \%)$, mordida cruzada anterior $(17,4 \%)$, sobresalte exagerado $(8,5 \%)$, sobremordida exagerada $(5,0 \%)$ y mordida abierta anterior (5,0\%). Se evidencia una alta prevalencia de maloclusiones y alteraciones ortodónticas en las comunidades nativas evaluadas, por lo que es necesario implementar programas preventivos para mejorar la salud bucal de estas poblaciones marginadas.
\end{abstract}

Palabras clave: Maloclusión; Salud bucal; Población indígena; Niño; Adolescente; Perú (fuente: DeCS BIREME).

\section{MALOCCLUSIONS IN CHILDREN AND ADOLESCENTS FROM VILLAGES AND NATIVE COMMUNITIES IN THE UCAYALI AMAZON REGION IN PERU}

\begin{abstract}
We conducted a cross-sectional descriptive study to assess the prevalence of malocclusions in children and adolescents aged 2 to 18 years of villages and native communities of the Ucayali jungle of Peru. We assessed the presence of malocclusions using Angle's classification and orthodontic changes. We evaluated 201 individuals, $106(52.7 \%)$ were women, most of them $(54.7 \%)$ had between 6 and 12 years. The prevalence of malocclusions was $85.6 \%$, the most prevalent according to Angle's classification was class I (59.6\%). Orthodontic alterations were present in $67.2 \%$ of cases. The most frequent were dental crowding $(28.4 \%)$, anterior crossbite $(17.4 \%)$, exaggerated overjet $(8.5 \%)$, excessive overbite $(5.0 \%)$ and anterior open bite $(5.0 \%)$. We found a high prevalence of malocclusion and orthodontic changes in the evaluated native communities, highlighting the need to implement preventive programs to improve the oral health of these negleted populations.
\end{abstract}

Keywords: Malocclusion; Oral health; population groups; Chid; Adolescent; Peru (source: MeSH NLM).

\section{INTRODUCCIÓN}

Las maloclusiones o problemas de oclusión dental, son el resultado de la adaptación de la región orofacial a varios factores etiológicos, resultando en diversas implicaciones que varían desde la insatisfacción estética hasta alteraciones en el habla, masticación, deglución, disfunciones temporomandibulares y dolor orofacial ${ }^{(1-4)}$. En la mayoría de los casos no hay un solo factor causal, sino que hay muchos interactuando y sobreponiéndose unos sobre otros. Sin embargo, se pueden definir dos componentes principales en su etiología, que son la predisposición genética y los factores exógenos o ambientales, que incluyen todos los elementos capaces de condicionar una maloclusión durante el desarrollo craneofacial ${ }^{(1,5-7)}$.

Según la Organización Mundial de la Salud, las maloclusiones constituyen la tercera mayor prevalencia entre las enfremedades bucales, después de caries

\footnotetext{
Facultad de Odontología, Universidad Nacional Mayor de San Marcos, Lima, Perú.

2 Departamento de Estomatología Preventiva y Social, Facultad de Odontología Universidad Nacional Mayor de San Marcos, Lima, Perú.

Facultad de Estomatología, Universidad Peruana Cayetano Heredia, Lima, Perú.

Facultad de Odontología, Universidad Inca Garcilaso de la Vega, Lima, Perú

a Cirujano Dentista; ${ }^{\mathrm{b}}$ Magíster en Estomatología, Doctorando en Estomatología; ${ }^{\mathrm{C}}$ Especialista en Estomatología Pediátrica.

* El resumen de esta investigación fue presentado en la XVII Reunión Anual de la International Association for Dental Research (IADR) - División Perú y IX Reunión Anual de la Asociación de Investigación Odontológica del Perú (AIOP), el 22 de octubre de 2010.
}

Recibido: 11-11-10 Aprobado: 09-03-11 
y enfermedad periodontal. En el Perú, tienen una prevalencia del $70 \%{ }^{(8,9)}$. El conocimiento de la situación epidemiológica de la población peruana es esencial para la implementación de programas que contemplen acciones preventivas, interceptivas y de tratamiento.

En el Perú se vienen realizando estudios epidemiológicos sobre maloclusiones desde $1954^{(6,10,11)}$, sobre todo en la capital peruana, existiendo algunos estudios en regiones de la costa, sierra y selva, con una prevalencia de $81,9 \%, 79,1 \%$ y $78,5 \%$ respectivamente. Siendo esta última región la que menos datos reportados presenta ${ }^{(10-}$ 14). Casi todos los estudios muestran datos de población urbana, especialmente aquellas de fácil acceso, pero existen muy pocos reportes de población rural y de comunidades nativas ${ }^{(13,14)}$.

Considerando que Perú es un país con una amplia diversidad racial, existen poblaciones excluidas y dispersas con características particulares que aún no han sido estudiadas. Por tal motivo, el objetivo de este estudio fue determinar la prevalencia de maloclusiones en niños y adolescentes de poblaciones excluidas y dispersas pertenecientes a diversos caseríos y comunidades nativas de la Amazonía de Ucayali, según sexo y edad.

\section{EL ESTUDIO}

Se realizó un estudio descriptivo transversal con 201 niños y adolescentes de 2 a 18 años de edad, sin antecedentes de tratamiento ortodóntico previo, seleccionados de un total de 372 niños y adolescentes de 2 a 18 años, pertenecientes a 18 caseríos y comunidades nativas (Purín, Señor de los Milagros, Nueva Generación, Buenos Aires, Bella Flor, Nuevo Putaya, Perlas del Imiria, Pacífico, Unión Vecinal, Nohaya, Nueva Yarina, Vinoncuro, Esperanza, Nazareth, Mahía, Tomahao, Jacaya y Putaya) del distrito de Masisea, Ucayali - Perú, a las cuales solo se tuvo acceso por vía fluvial. Las personas examinadas fueron todos los que acudieron a la atención integral de salud, realizada en cada uno de los caseríos y de las comunidades nativas, como parte del Programa AISPED-Masisea (Atención Integral de Salud a Poblaciones Excluidas y Dispersas). En su mayoría la población estudiada, no contaba con establecimientos de salud, y los pocos lugares que sí lo tenían no contaban con un odontólogo. Es por esto que la única atención odontológica que reciben es por medio de este programa. El nivel socioeconómico era bajo y las condiciones de vida variaban de muy pobre a extrema pobreza. La alimentación provenía de vegetales y animales producidos en sus tierras, ya que el consumo de productos importados era muy costoso. La educación que tenían con respecto a salud bucal era mínima, hasta a veces nula, por ello los problemas dentales, en su mayoría, terminaban en extracción dental.

Previo al estudio, se obtuvo la aprobación del Jefe del Programa, quien dirigía el viaje, además se obtuvo la autorización de los padres de los niños involucrados en el estudio. La evaluación fue realizada por un mismo cirujano dentista en los distintos lugares, bajo luz natural, con visión directa y con la ayuda de un bajalenguas en el área común de atención dental. Se registró: edad, sexo, maloclusión según la clasificación de Angle y maloclusión según la presencia de las siguientes alteraciones ortodónticas: apiñamiento dentario (desplazamiento $\geq 1$ mm entre los puntos de contacto de los dientes), mordida cruzada anterior y mordida cruzada posterior (dientes con relación vestíbulo lingual anormal), sobresalte exagerado ( $\geq 3,5 \mathrm{~mm}$ ), sobremordida exagerada $(\geq 3,5$ $\mathrm{mm})$ y mordida abierta anterior $(\geq 1 \mathrm{~mm})^{(2)}$.

Para la determinación de la maloclusión según Angle, fueron adoptados sus criterios. Oclusión normal: cuando existe relación normal entre molares, dientes alineados y una línea de oclusión con una leve curvatura. Maloclusión clase I: relaciones molares normales, pero línea de oclusión incorrecta por malposición dentaria, rotaciones u otras causas. Maloclusión clase II: molar inferior posicionada distalmente en relación a la molar superior, línea de oclusión inespecífica. Maloclusión clase III: molar inferior posicionada mesialmente en relación a la molar superior, línea de oclusión inespecífica ${ }^{(1,4,15)}$. Para la determinación de las alteraciones ortodónticas fueron adoptados los criterios del Componente de Salud Dental (Dental Health Component - DHC) del Índice de Necesidad de Tratamiento Ortodóntico (Index of Orthodontic Treatment Need-IONT) ${ }^{(2,16)}$.

Los datos recolectados fueron analizados en el programa SPSS 15.0. Los resultados se presentaron de forma descriptiva, a partir de sus frecuencias absolutas y relativas.

\section{HALLAZGOS}

De los 372 niños y adolescentes entre 2 y 18 años moradores de los distintos lugares visitados, solo 201 $(54,0 \%)$ acudieron a la atención brindada por AISPED, $106(52,7 \%)$ fueron mujeres, según grupo etario, la mayoría (110) tuvieron entre 6 y 12 años, seguido por el grupo de 2 a 5 años (55) y fueron menos los de 13 a 18 años (36).

Al evaluar la clasificación de Angle (Tabla 1) solo se consideró los grupos etarios de 6-12 años y de 13- 
Tabla 1. Maloclusión de Angle según sexo y grupo etario en niños y adolescentes de 6 a 18 años de comunidades nativas de la Amazonía peruana.

\begin{tabular}{|c|c|c|c|c|c|c|c|c|c|c|}
\hline \multirow{3}{*}{ Clasificación de Angle } & \multirow{2}{*}{\multicolumn{2}{|c|}{$\begin{array}{c}\text { Total } \\
(n=146)\end{array}$}} & \multicolumn{4}{|c|}{ Género } & \multicolumn{4}{|c|}{ Grupo etario } \\
\hline & & & \multicolumn{2}{|c|}{$\begin{array}{c}\text { Masculino } \\
(n=70)\end{array}$} & \multicolumn{2}{|c|}{$\begin{array}{c}\text { Femenino } \\
(n=76)\end{array}$} & \multicolumn{2}{|c|}{$\begin{array}{c}\text { 6-12 años } \\
(n=110)\end{array}$} & \multicolumn{2}{|c|}{$\begin{array}{c}\begin{array}{c}13-18 \text { años } \\
(n=36)\end{array}\end{array}$} \\
\hline & $\mathbf{n}$ & $(\%)$ & $\mathbf{n}$ & (\%) & $\mathbf{n}$ & $(\%)$ & $\mathbf{n}$ & (\%) & $\mathbf{n}$ & $(\%)$ \\
\hline Oclusión normal & 21 & $(14,4)$ & 6 & $(8,6)$ & 15 & $(19,7)$ & 11 & $(10,0)$ & 10 & $(27,8)$ \\
\hline Maloclusión clase I & 87 & $(59,6)$ & 42 & $(60,0)$ & 45 & $(59,2)$ & 64 & $(58,2)$ & 23 & $(63,9)$ \\
\hline Maloclusión clase II & 27 & $(18,5)$ & 18 & $(25,7)$ & 9 & $(11,8)$ & 24 & $(21,8)$ & 3 & $(8,3)$ \\
\hline Maloclusión clase III & 11 & $(7,5)$ & 4 & $(5,7)$ & 7 & $(9,2)$ & 11 & $(10,0)$ & 0 & $(0,0)$ \\
\hline
\end{tabular}

18 años de edad encontrándose que la mayor parte de individuos (125 - 85,6\%), presentó algún tipo de maloclusión, siendo la más frecuente la maloclusión clase I con 87 individuos $(59,6 \%)$, seguida por la maloclusión clase II con 27 individuos (18,5\%). La maloclusión clase I fue más frecuente tanto en el sexo masculino como en el femenino con $42(60,0 \%)$ y 45 individuos $(59,2 \%)$ respectivamente. La clase III de Angle fue la que se encontró con menor frecuencia en ambos sexos con $4(5,7 \%)$ y 7 individuos $(9,2 \%)$ para el sexo masculino y femenino respectivamente. Según el grupo etario, la clase I de Angle fue más prevalente en ambos grupos con $64(58,2 \%)$ y $23(63,9 \%)$ individuos para el grupo de 6-12 años y 13-18 años respectivamente. La menos frecuente fue la clase III de Angle con $11(10 \%)$ y ningún (0\%) individuos para los grupos de 6-12 años y 13-18 años respectivamente.

La Tabla 2 presenta la prevalencia de alteraciones ortodónticas evaluadas, además de la distribución según sexo y grupo etario. Las alteraciones ortodónticas más frecuentes fueron apiñamiento dentario, mordida cruzada anterior y sobresalte exagerado. En ambos sexos fue más frecuente la presencia de apiñamiento dentario. En el grupo etario de 2 a 5 años fue más frecuente la mordida cruzada anterior mientras que en los grupos de 6 a 12 años y de 13 a 18 años fue el apiñamiento dentario.

\section{DISCUSIÓN}

Existe una evidente falta de información sobre la prevalencia de maloclusiones en poblaciones alejadas, debido a que las investigaciones se han preocupado principalmente de la situación de salud de áreas metropolitanas. Estudios de prevalencia de maloclusiones, como el presente, son poco comunes por la dificultad para el acceso a estas zonas. Sin embargo, son de vital importancia ya que nos brindan datos de poblaciones no estudiadas hasta la actualidad. Si bien es cierto, el tipo de muestreo realizado fue no probabilístico y se trata de una muestra no representativa, no obstante, es amplia con respecto a la población, lo que la convierte en un estudio epidemiológico de interés y relevancia.

La prevalencia de maloclusiones $(85,6 \%)$ en este estudio fue mayor a la encontrada anteriormente $(78,5 \%)$ para zonas geográficas similares ${ }^{(12-14)}$, así como para otras zonas geográficas como costa y sierra ${ }^{(7,12)}$. Según la clasifica Angle, la maloclusión clase I sigue siendo la que tiene mayor prevalencia (59,6\%) así como lo indican estudios anteriores en población peruana; sin embargo, se observa una disminución de este porcentaje en los últimos 10 años ${ }^{(6,7,10-14)}$. Las maloclusiones según alteración ortodóntica con mayor prevalencia fueron: apiñamiento dentario, mordida cruzada anterior y sobresalte exagerado, encontrándose el apiñamiento

Tabla 2. Alteraciones ortodónticas según sexo y grupo etario en niños y adolescentes de 2 a 18 años de comunidades nativas de la Amazonía peruana.

\begin{tabular}{|c|c|c|c|c|c|c|c|c|c|c|c|c|}
\hline \multirow{3}{*}{ Alteraciones ortodónticas } & \multirow{2}{*}{\multicolumn{2}{|c|}{$\begin{array}{c}\text { Total } \\
(\mathrm{N}=201)\end{array}$}} & \multicolumn{4}{|c|}{ Sexo } & \multicolumn{6}{|c|}{ Grupo etario } \\
\hline & & & \multicolumn{2}{|c|}{$\begin{array}{c}\text { Masculino } \\
(n=95)\end{array}$} & \multicolumn{2}{|c|}{$\begin{array}{c}\text { Femenino } \\
(n=106)\end{array}$} & \multicolumn{2}{|c|}{$\begin{array}{c}2-5 \text { años } \\
(n=55)\end{array}$} & \multicolumn{2}{|c|}{$\begin{array}{c}\text { 6-12 años } \\
(n=110)\end{array}$} & \multicolumn{2}{|c|}{$\begin{array}{c}\text { 13-18 años } \\
(n=36)\end{array}$} \\
\hline & $\mathbf{n}$ & $(\%)$ & $\mathbf{n}$ & $(\%)$ & $\mathrm{n}$ & $(\%)$ & $\mathbf{n}$ & $(\%)$ & $\mathrm{n}$ & $(\%)$ & $\mathbf{n}$ & $(\%)$ \\
\hline Apiñamiento dentario & 57 & $(28,4)$ & 26 & $(27,4)$ & 31 & $(29,2)$ & 0 & (0) & 38 & $(34,5)$ & 19 & $(52,8)$ \\
\hline Mordida cruzada anterior & 35 & $(17,4)$ & 21 & $(22,1)$ & 14 & $(13,2)$ & 9 & $(16,4)$ & 25 & $(22,7)$ & 1 & $(2,8)$ \\
\hline Mordida cruzada posterior & 6 & $(3,0)$ & 3 & $(3,2)$ & 3 & $(2,8)$ & 1 & $(1,8)$ & 4 & $(3,6)$ & 1 & $(2,8)$ \\
\hline Sobresalte exagerado & 17 & $(8.5)$ & 13 & $(13,7)$ & 4 & $(3,8)$ & 0 & $(0,0)$ & 15 & $(13,6)$ & 2 & $(5,6)$ \\
\hline Sobremordida exagerada & 10 & $(5,0)$ & 5 & $(5,3)$ & 5 & $(4,8)$ & 0 & $(0,0)$ & 9 & $(8,2)$ & 1 & $(2,8)$ \\
\hline Mordida abierta anterior & 10 & $(5,0)$ & 3 & $(3,2)$ & 7 & $(6,6)$ & 0 & $(0,0)$ & 8 & $(7,3)$ & 2 & $(5,6)$ \\
\hline Sin alteraciones & 66 & $(32,8)$ & 24 & $(25,3)$ & 42 & $(39,6)$ & 45 & $(81,2)$ & 11 & $(10)$ & 10 & $(27,8)$ \\
\hline
\end{tabular}


dentario y el sobresalte exagerado con mayor frecuencia en el sexo masculino y en el grupo etario de 6 a 12 años y la mordida cruzada anterior en el sexo femenino y en el grupo etario de 2 a 5 años. La indicación del tratamiento temprano para algunas de las maloclusiones evaluadas, resalta la importancia de haber evaluado individuos con un amplio rango etario (2-18 años).

Cuando existe un desequilibrio entre el desgate dentario interproximal fisiológico (dado por el tipo de alimentación) y el componente anterior de fuerzas de la oclusión, el crecimiento de los maxilares se ve limitado. La alta prevalencia de alteraciones ortodónticas puede deberse a la alimentación basada en comidas blandas, no pudiendo contrarrestarse el efecto del componente anterior de fuerzas apareciendo así apiñamiento dentario y protrusión dentaria, entre otras alteraciones.

La mayoría de estudios de prevalencia de maloclusiones muestran altos porcentajes como se observa en la Tabla 3. Debido a que un gran número de niños presentan algún tipo de maloclusión, es importante resaltar que no implica necesariamente una necesidad de tratamiento ortodóntico. Una misma maloclusión puede presentar diferentes niveles de gravedad las cuales reciben diferentes prioridades de tratamiento, especialmente en salud pública, donde la demanda de servicios es mayor que la oferta ${ }^{(17)}$.

En Perú, existen muchas poblaciones excluidas y dispersas para las cuales el Sistema de Salud Pública no asiste problemas de oclusión y como todas estas poblaciones dependen exclusivamente de este sistema, se entiende que muchos pacientes portadores de maloclusiones no están siendo atendidos. Cabe resaltar la importancia de levantamientos epidemiológicos como el presente, que colocan a las maloclusiones como un problema importante de salud bucal, buscando promover la oferta de servicios ortodónticos preventivos e interceptivos, para esta parte de población peruana no asistida.

Tabla 3. Algunos estudios de prevalencia de maloclusión.

\begin{tabular}{|c|c|c|c|c|}
\hline Autor & País & Muestra & $\begin{array}{l}\text { Edad } \\
\text { (años) }\end{array}$ & $\begin{array}{c}\text { Maloclusión } \\
(\%)\end{array}$ \\
\hline Ciuffolo et al. ${ }^{(18)}$ & Italia & 810 & $11-14$ & 93,0 \\
\hline Thilander et al. (19) & Colombia & 4724 & $5-17$ & 88,0 \\
\hline Behbehani et al. (20) & Kuwait & 1299 & $13-14$ & 86,3 \\
\hline Mills (21) & EEUU & 1455 & $8-18$ & 82,5 \\
\hline Brito et al. (2) & Brasil & 407 & $9-12$ & 80,8 \\
\hline Ng'ang'a et al. (22) & Kenya & 919 & $13-15$ & 72,0 \\
\hline Saleh ${ }^{(23)}$ & Líbano & 851 & $9-15$ & 59,7 \\
\hline Drummond (24) & Sudáfrica & 6918 & 12 & 52,3 \\
\hline
\end{tabular}

Se concluye que los niños y adolescentes de 2 a 18 años de edad de caseríos y comunidades nativas de la selva de Ucayali evaluados en este estudio presentan una elevada prevalencia de maloclusiones, siendo la más frecuente la clase I, en algo más de la mitad de la muestra en ambos sexos y en ambos grupos etarios de 6-12 años y de 13-18 años; las alteraciones ortodónticas frecuentes fueron: el apiñamiento dentario, la mordida cruzada anterior y el sobresalte exagerado. En ambos sexos y en los grupos etarios de 6-12 años y de 1318 años fue más frecuente el apiñamiento dentario, en el grupo etario de 2-5 años fue más prevalente la mordida cruzada anterior. Debido a la gran presencia de maloclusiones se hace necesario este tipo de estudios, para que el Ministerio de Salud pueda planear y ejecutar tratamientos ortodónticos preventivos e interceptivos así como otros procedimientos con el objetivo de obtener un ambiente bucal favorable para el desarrollo normal de la oclusión.

\section{Fuentes de Financiamiento}

Autofinanciado.

\section{Conflictos de Interés}

Los autores declaran no tener conflictos de interés en la publicación del presente artículo.

\section{REFERENCIAS BIBLIOGRÁFICAS}

1. Proffit WR. Ortodoncia contemporánea: teoría y práctica. $3^{\text {ra }}$ ed. Madrid: Elsevier; 2001.

2. Brito DI, Dias PF, Gleiser R. Prevalência de más oclusões em crianças de 9 a 12 anos de idade da cidade de Nova Friburgo (Rio de Janeiro). R Dental Press Ortodon Ortop Facial. 2009;14(6):50-7.

3. Albuquerque SSL, Duarte RC, Cavalcanti AL, Beltrão EM. Prevalência de más oclusões em crianças com 12 a 36 meses de idade em João Pessoa, Paraíba. R Dental Press Ortodon Ortop Facial. 2009;14(6):118-24.

4. Vellini F. Ortodoncia: diagnóstico y planificación clínica. Sao Paulo: Editorial Las Artes Médicas; 2002.

5. Almeida RR, Almeida PRR, Almeida MR, Garib DG, Almeida PC, Pinzan A. Etiologia das más oclusões: causas hereditárias e congênitas, adquiridas gerais, locais e proximais (hábitos bucais). Rev Dent Press Ortodon Ortop Maxilar. 2000;5(6):107-29.

6. Menéndez LV. Clasificación de la maloclusión según Angle en el Perú. análisis de 27 trabajos de investigación. Odontol Sanmarquina. 1998;1(2):e10.

7. Salazar NP. Prevalencia de maloclusiones en niños escolares del departamento de Tumbes. [Tesis bachiller]. Lima: Facultad de Odontología, Universidad Nacional Mayor de San Marcos; 2003.

8. Pan American Health Organization. Health in the Americas. Washington DC: PAHO; 1998. p. 413-27. 
9. Pan American Health Organization. Ten-year evaluation of regional core health data initiative. Epidemiol Bull PAHO. 2004;25(3):1-16.

10. Koenig A. Estudio de la prevalencia de las maloclusiones en 100 escolares de la ciudad de Iquitos. [Tesis bachiller]. Lima: Facultad de Odontología, Universidad Nacional Mayor de San Marcos; 1954.

11. Orellana O, Mendoza J, Perales S, Marengo H. Estudio descriptivo de todas las investigaciones sobre maloclusiones realizadas en las universidades de Lima, Ica y Arequipa. Odontol Sanmarquina. 2000;1(5):e8.

12. Montalvan H. Frecuencia de maloclusiones dentarias en los escolares del oriente peruano, Iquitos. [Tesis bachiller]. Lima: Facultad de Odontología, Universidad Nacional Mayor de San Marcos; 1961.

13. Chu C. Prevalencia de maloclusiones en individuos de 12 a 18 años en la comunidad nativa de San Antonio de Sonomoro, provincia Satipo. [Tesis bachiller]. Lima: Universidad Peruana de Cayetano Heredia; 1988.

14. Kohatsu D. Prevalencia de maloclusiones en individuos de 12 a 18 años de edad pertenecientes a la población rural de la provincia de Satipo, Junín. [Tesis bachiller]. Lima: Universidad Peruana de Cayetano Heredia; 1988.

15. Angle EH. Classification of malocclusion. Dental Cosmos. 1899;41(3):248-64.

16. Brook PH, Shaw WC. The development of an index of orthodontic treatment priority. Eur J Orthod. 1989;11(3):309-20.

17. Marques LS, Barbosa CC, Ramos-Jorge ML, Pordeus IA, Paiva SM. Prevalência da má oclusão e necessidade de tratamento ortodôntico em escolares de 10 a 14 anos de idade em Belo Horizonte, Minas Gerais, Brasil: enfoque psicossocial. Cad Saude Publica. 2005;21(4):1099-106.
18. Ciuffolo F, Manzoli L, Dattilio M, Tecco S, Muratore F, Festa $F$, et al. Prevalence and distribution by gender of occlusal characteristics in a sample of Italian secondary school students: A cross-sectional study. Eur J Orthod. 2005;27(6):601-6.

19. Thilander B, Pena L, Infante C, Parada SS, de Mayorga C. Prevalence of malocclusion and orthodontic treatment need in children and adolescents in Bogota, Colombia. An epidemiological study related to different stages of dental development. Eur J Orthod. 2001;23(2):153-67.

20. Behbehani F, Artun J, Al-Jame B, Kerosu H. Prevalence and severity of malocclusion in adolescent Kuwaitis. Med Princ Pract. 2005;14(6):390-5.

21. Mills LF. Epidemiologic studies of occlusion. IV. The prevalence of malocclusion in a population of 1,455 school children. J Dent Res. 1966;45(2):332-36.

22. Ng'ang'a, PM, Ohito F, Ogaard B, Valderhaug J. The prevalence of malocclusion in 13 to 15 -year-old children in Nairobi, Kenya. Acta Odontol Scand. 1996;54(2):126-30.

23. Saleh FK. Prevalence of malocclusion in a sample of Lebanese schoolchildren: An epidemiological study. East Mediterr Health J. 1999;5(2):337-43.

24. Drummond RJ. Orthodontic status and treatment need of 12-year-old children in South Africa: An epidemiological study using the Dental Aesthetic Index. 2003. [Tesis Maestría]. Pretoria: School of Dentistry, University of Pretoria; 2003.

Correspondencia: Arón Aliaga- Del Castillo.

Dirección: Jr. J.J. Pasos 490 Int. 401, Lima 21, Perú.

Teléfono: 332-4318; 996-845677.

Correo electrónico: a_aliaga@hotmail.com

\section{FE DE ERRATAS:}

Rev Peru Med Exp Salud Publica. 2010; 27(2): 233

Dice:

Cabe mencionar que esta restricción, podría, en cierto grado, explicar el descenso de las agresiones, homicidios y suicidios, lo que correlacionó con la fracción de día de expendio de licores observados en La Victoria ${ }^{(18)}$.

Debe decir:

Cabe mencionar que esta restricción, podría, en cierto grado, explicar el descenso de las agresiones, homicidios y suicidios, lo que correlacionó con la fracción de día de expendio de licores observados en La Victoria (17).

Rev Peru Med Exp Salud Publica. 2010; 27(4): 613

Dice:

Pedro E. Huapaya ${ }^{2}$

2 Dirección Regional de Salud, Ministerio de Salud, Lima, Perú.

Debe decir:

Pedro E. Huapaya²

${ }^{2}$ Dirección Regional de Salud-Lima, Gobierno Regional de Lima, Perú. 